Bioscientia Medicina: Journal of Biomedicine \& Translational Research

Journal Homepage: www.bioscmed.com

\title{
Condylomata Acuminata in Adolescent Girl 19th Years Old Successful with Multimodality Therapy and Combination with HPV Vaccination: A Case Report
}

\section{Dwi Sabtika Julia ${ }^{*}$, Qaira Anum², Rina Gustia ${ }^{3}$}

1,2,3 Dermatology and Venereology Department, Faculty of Medicine, Andalas University/Dr. M Djamil Hospital, Padang, Indonesia

\section{A R T I C L E I N F O \\ Keywords: \\ Condylomata Acuminata \\ Human Pappilloma Virus (HPV) \\ *Corresponding author: \\ Dwi Sabtika Julia \\ E-mail address: \\ dwisabtikajulia@gmail.com}

All authors have reviewed and approved the final version of the manuscript.

https://doi.org/10.32539/bsm.v5i10.408

\begin{abstract}
A B S T R A C T
Background: Condylomata acuminata $(\mathrm{KA})$ or better known as genital warts disease is a genital area infectious disease caused by Human papilloma virus (HPV). The highest prevalence infection of the condylomata acuminata occurs during active sexual periods, namely age 17-33 years, with the peak occurring at the age of 20-24 years. Currently HPV 16 and 18 are known to cause malignancy in the genital and anogenital region including cervical cancer and anal cancer, while HPV 6 and 11 are the cause of $90 \%$ of condylomata acuminata. HPV is linked to the findings of 500,000 new cases of cervical cancer and 250,000 deaths from cervical cancer each year worldwide. 1 Case report: One case of condylomata acuminata in a 19-year-old adolescent girl with a major complaint of warts around her genitals that felt itchy and gradually getting bigger since 2 weeks ago. Patient was a student and unmarried. History of sexual contact with men with genito-genital without using condoms since 5 months ago. Venereological state : on the vulva and perineal there were multiple vegetations with verucose surfaces with the largest size $0.8 \mathrm{~cm} \times 0.5 \mathrm{~cm} \times 0.1 \mathrm{~cm}$ and the smallest size $0.2 \mathrm{~cm} \times 0.2 \mathrm{~cm} \times 0.1 \mathrm{~cm}$. Acetowhite examination result was positive and PCR examination results found positive HPV types 6,11,16 and 18 . Discussion: Patients are diagnosed to condylomata acuminata with a history of free sex at an early age. The condylomata acuminata experienced by patients was type $6,11,16$ and 18 . These types of 16 and $18 \mathrm{HPV}$ are know to cause malignancy in the genital and anogenital region including cervical cancer and anal cancer.
\end{abstract}

\section{Introduction}

Genital condylomata acuminata or better known as genital wart disease is an infection of the genital area in the form of papillomatosis with a verrucous surface caused by the Human papilloma virus (HPV). ${ }^{1}$

This problem arises because most people do not know that this virus can spread easily through sexual contact and HPV infection is often asymptomatic. Primary HPV transmission occurs through skin to skin contact due to micro-abrasion in the epidermis during sexual contact. HPV infection has an incubation period of 3 weeks until 8 months., 80\% was benign and can heal spontaneously, but $20 \%$ of cases of HPV infection can experience persistence and have the potential become malignant. ${ }^{2}$

Risk factors for malignancy by HPV infection include the number of sexual partners, age at first sexual intercourse, persistent HPV infection, impaired immune cell mediated system and genetic factors. Some studies show that the number of sex partners is more than one and the early age do the first sexual intercourse increases the risk of contracting HPV infection. Bachtiary et al (2002) and van der Graaf et al (2002) stated that multiple HPV infection was associated with an increased risk of disease progression. ${ }^{5}$

The diagnosis of condyomata acuminata can be made based on history, clinical, and physical examination. In doubtful cases, an examination can be carried out among others: acetic acid test, colposcopy, histopathological examination and PCR 
examination. PCR is a method that is often used and very sensitive to detect DNA viruses. ${ }^{6}$

The main reason for treatment of genital warts is to improve symptoms (including eliminating cosmetic problems) and finally, removing warts. In most patients, treatment can induce a period of free warts. If left untreated, visible genital warts can heal on their own, the size remains or does not experience improvement or the size and amount will increase and become larger. Available therapies for genital warts are likely to reduce the warts, but may not eradicate of infectivity HPV. CDC recommendation therapy for condylomata acumiata is cryotherapy using liquid nitrogen or cryoprobe repetition application every 1-2 weeks, or $10 \%-25 \%$ podophylline resin in a solution of benzoin, or trichloroacetic acid (TCA) 80\% - $90 \%$, or surgical removal either by excision of tangential scissors, excision of tangential shaving, curettage, or electrical surgery. ${ }^{7,8}$

Recurrence after treatment often occurs especially in patients with immunocompromised. A study showed that recurrence of genital and anogenital warts was found to be $12.9 \%$ in people with HIV infection compared to patients without HIV infection of $9.3 \%$. In cases with type 16 and $18 \mathrm{HPV}$ that are positive, potentially oncogenic and most often can cause cervical cancer. Therefore, early detection and management is very important, especially in high risk populations such as do routine pap smears and vaccinations. 9

\section{Case Report}

A female patient, 19 years old, came to the dermatology and venereology outpatient clinic on Dr. M Djamil Hospital Padang on May 8th 2019 with warts that grow around the genital that felt a little itchy and gradually getting bigger since 2 weeks ago. Initially 1 month ago, there was warts on her genital, skincolored, small warts as green beans size. The warts sometimes itchy, and increase in size and more multiple since \pm 2 weeks ago. This was the first time she complained of having warts on her genitals. Patients often scratch the warts on her genital using hands because the warts sometimes itchy.

Patients are students and unmarried. There was a history of genito-genital sexual contact with men (with her boyfriend, her senior on campus) without using condom since 5 months ago. The last time was having sexual intercourse 3.5 months ago with genito-genital sexual contact without using a condom with her boyfriend. History of sexual contact of her sexual partners with another women and men except the patients was unknown. The patient did not know whether her sexual partner had warts or complaints of discharge from the genitals or genital ulcers.

On physical examination, vital sign and another physical examination was in normal limit. On venereological state, from inspection on vulva was found multiple vegetation more than 5 warts with a verrucous surface with the largest size $1.4 \mathrm{~cm} \times 0.8 \mathrm{~cm}$ $\mathrm{x} 0.2 \mathrm{~cm}$ and the smallest size $0.2 \mathrm{~cm} \times 0.2 \mathrm{~cm} \times 0.1$ $\mathrm{cm}$. On Perineal found multiple vegetation $(+)$ more than 5 warts with a verrucous surface with the largest size $0.8 \mathrm{~cm} \times 0.5 \mathrm{~cm} \times 0.2 \mathrm{~cm}$ and the smallest size 0.2 $\mathrm{cm} \times 0.1 \mathrm{~cm} \times 0.1 \mathrm{~cm}$. Inguinal medial lymphnodes no enlargement. And we can't examination with inspeculo because the patient unmarried. Acetowhite test was positif.

From Laboratory Examination Result was normal limit. Haemoglobine $13 \mathrm{~g} / \mathrm{dl}$, hematocrite $40 \%$, platelet $252.000 / \mu \mathrm{L}$, WBC $8.970 / \mu \mathrm{L}$, PT 10.0 second, APTT 31.3 second, CD4 Absolut 683 sel / $\mu \mathrm{L}, \mathrm{CD} 4 \% 36.3$ \%, CD8 Absolut 688 sel / $\mu \mathrm{L}, \mathrm{CD} 8 \% 36.6 \%$, Ratio of CD4 : CD8 0.99, VDRL non reactive, TPHA non reactive and HIV non reactive.

We did PCR Examination in this patient with result detection of human papilloma Virus (HPV) was positive HPV type 6, 11, 16 and 18. From anamnesis, physical examination, venereological state, vaginal discharge examination, laboratory examination and PCR examination, we diagnose this patient with Condylomata Acuminata Genital.

General treatment for this patient were explain to the patient about her disease, the genital and perineal warts of the patient causes by a virus, which is one of the HPV viruses that can be transmitted through with sexual intercourse. Explain to the patient about choices of treatment we can do to the patient, how to do it and the side effects of the treatment and explain 
the possibility of recurrence. Advise the patient to bring transmitted diseases. Don't do free sex again. Regular control to the IMS Division of the dermatology and venereology outpatient clinic of Dr. M Djamil Hospital, Padang. Advice the patient to do vaccination of HPV.

Specific Treatment we did to this patient were chemosurgery with TCA $80 \%$ in the genital and perineal warts every week (application of vaseline in the area around TCA $80 \%$ bottling), cryosurgery in the genital warts on upper side of labia mayora sinistra, and Electrosurgery or electrocauter on warts in pubis, both of groin and upper side of labia mayora sinistra.

For the first treatment we did a chemosurgery with $80 \%$ TCA for the treatment of her condylomata acuminata. After two times treatment with $80 \%$ TCA, the warts in perineal has been released but in the place of the warts appear erotion and ulceration with pussy. And then we treated this patient with Clindamycin twice a day $300 \mathrm{mg}$ p.o for 7 day and fusidic acid $2 \%$ cream twice a day applied in erotion around perineal area. Then, the erotion and ulceration around of perineal's area have begun to improve and dry out with no pus

Beside that, we did cryosurgery treatment on other location of warts, we did only one time cryosurgery in the genital warts on upper side of labia mayora sinistra. Three days after cryosurgery, appear blister in cryosurgery location (in upper side of labia mayora sinistra). Ten days after cryosurgery, appear blister in her sexual partners to do screening for sexually cryosurgery location (in the upper side of labia mayora sinistra) but in the lower side of labia mayora dextra didn't appear blister. And in the 17 day after cryosurgery, the warts in the upper side of labia mayora sinistra have mostly fallen out and one of the genital warts persists and didn't release but in the lower side of labia mayora sinistra area was persist and no changes.

And then 2 week later, there were appear a new warts in pubis and both of groin. On venereologycal state on pubic found multiple vegetation $(+)$ more than 5 warts with verrucous surface with the biggest size 0.4 $\mathrm{cm} \times 0,2 \mathrm{~cm} \times 0,2 \mathrm{~cm}$ and the smallest size $0,2 \mathrm{~cm} \mathrm{x}$ $0,1 \mathrm{~cm} \times 0,1 \mathrm{~cm}$. And we treated the patient with electrosurgery on warts in pubis, both of groin and upper side of labia mayora sinistra. There warts on pubis and both of groin has been released after this treatment.

Beside all kinds of treatment, the patient also had vaccinated HPV with Quadrivalent Vaccine (Gardasil ${ }^{\circledR}$ ). And one month, after the patient had complete there times vaccinated HPV with Quadrivalent Vaccine (Gardasil ${ }^{\mathbb{R}}$ ), all the wart has been released and didn't appear again. The Prognosis of this patient, Quo ad vitam was bonam, Quo ad sanationam was dubia ad bonam, Quo ad cosmeticum was dubia ad bonam and Quo ad functionam was dubia ad bonam.

Picture before acetowhite test and before treatment
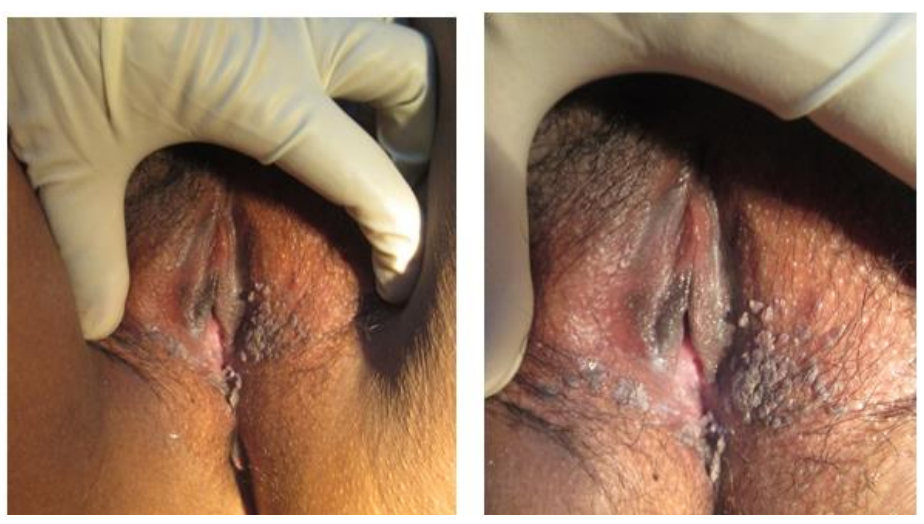

Figure 1. Condyloma acuminata anogenital on vulva found multiple vegetation more than 5 warts with a verrucous surface with the largest size $1.4 \mathrm{~cm} \times 0.8 \mathrm{~cm} \times 0.2 \mathrm{~cm}$ and the smallest size $0.2 \mathrm{~cm} \times 0.2 \mathrm{~cm} \times 0.1 \mathrm{~cm}$. On perineal found multiple vegetation more than 5 warts with a verrucous surface with the largest size $0.8 \mathrm{~cm} \times 0.5 \mathrm{~cm} \times 0.2 \mathrm{~cm}$ 
and the smallest size $0.2 \mathrm{~cm} \times 0.1 \mathrm{~cm} \times 0.1 \mathrm{~cm}$. This is a picture before acetowhite test and before treatment.

Picture after acetowhite test

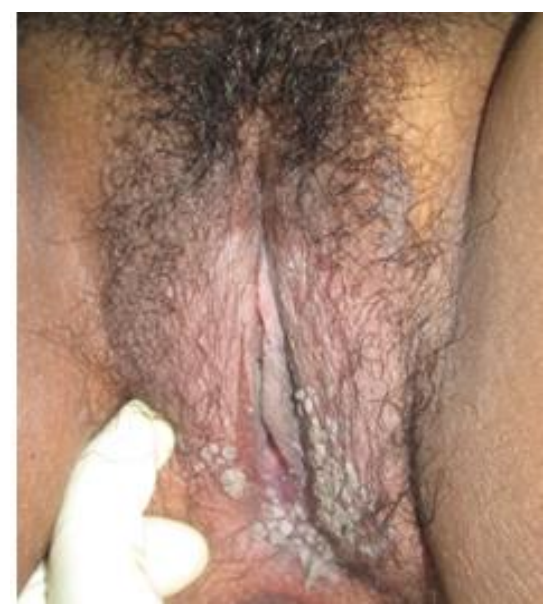

Figure 2. Condyloma acuminata anogenital on vulva and perineal after acetowhite test, all the wart was a change colour became white and the acetowhite was positive.

Picture after chemosurgery and cryosurgery
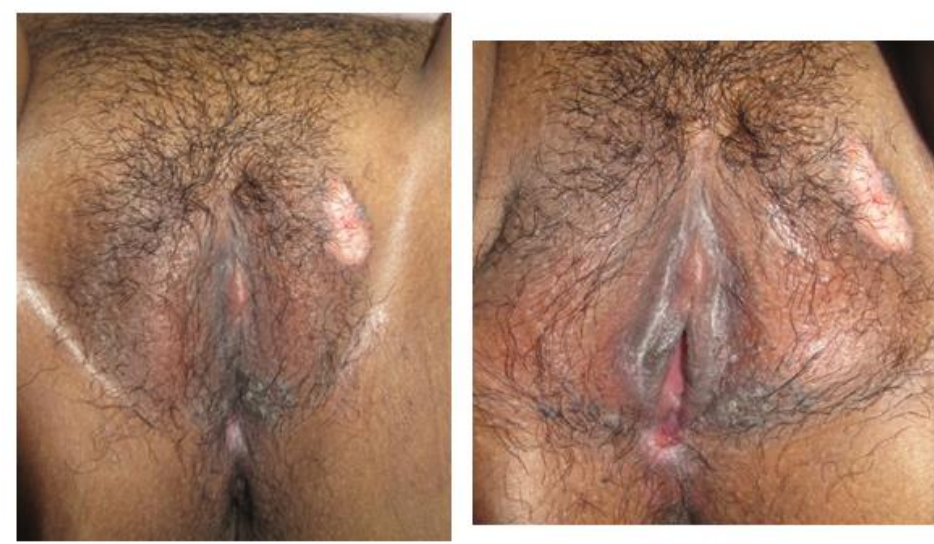

Figure 3. After two times treatment with TCA $80 \%$, the warts in perineal was clearance, but in the place of the warts appear erotion and ulceration. And after cryosurgery, most of the warts in the upper side of labia mayora sinistra was clearance but one of the genital warts persists and didn't clearance but in the lower side of labia mayora sinistra area was persist and no changes.

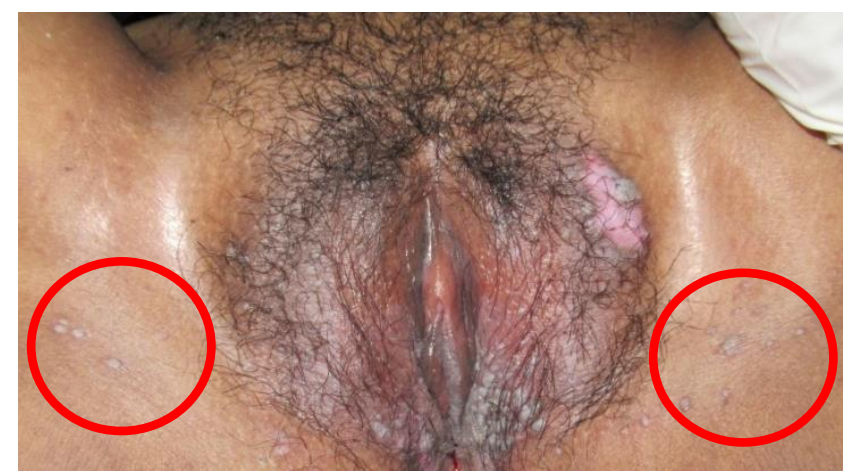

Figure 4. The red circle were a the location of electrosurgery treatment 
Picture 2 month later, the lesion was recurrence
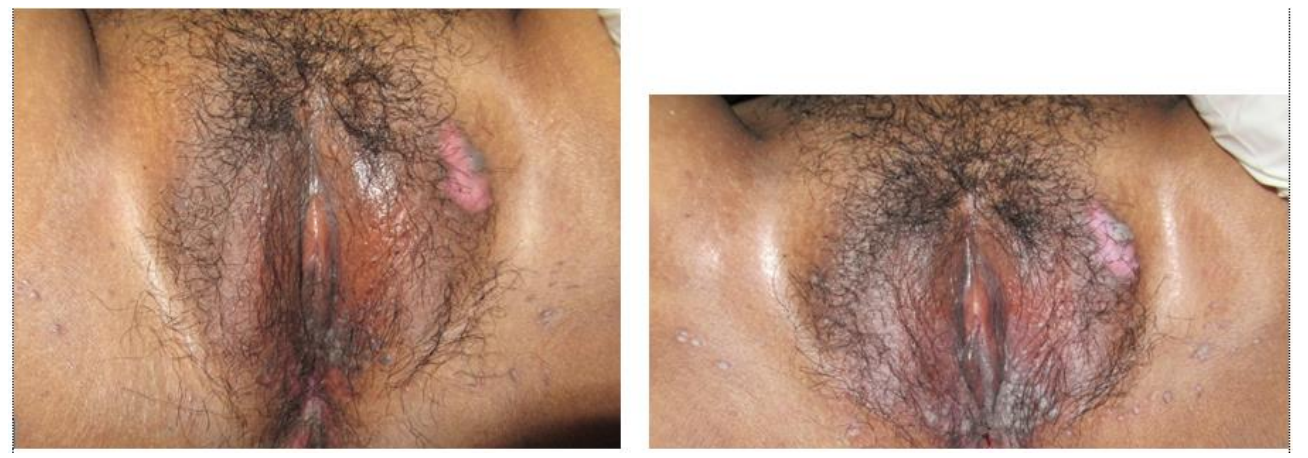

Figure 4. After 2 months later, appear a new warts in pubis and both of groin. On pubic found multiple vegetation more than 5 warts with verrucous surface with the biggest size $0,4 \mathrm{~cm} \times 0,2 \mathrm{~cm} \times 0,2 \mathrm{~cm}$ and the smallest size 0,2 $\mathrm{cm} \times 0,1 \mathrm{~cm} \times 0,1 \mathrm{~cm}$

\section{Discussion}

We presented a case of genital and perineal condylomata acuminata in adolescent girl $19^{\text {th }}$ years old with type of HPV low risk and high risk. Diagnosis in this case was made based on anamnesis, physical examination, laboratory examination and PCR examination.

Diagnosis of Condylomata Acuminata was based on clinical features. Doubtful lesions can be examined by acetowhite test in suspected lesions and waited for 10 until 15 minutes. This examination will entrust the arrangement with white discoloration in the area that supports HPV so that it can also be used for protection with KA, although it may be non-specific. Biopsy is not necessary and only be indicated if atypical lesions and diagnosis are doubtful, the lesion does not show response to standard therapy or if the disease worsens during administration of therapy.4,6

In this cases, acetowhite examination showed positive results and non-reactive VDRL and TPHA examinations. Histopathological examination was not carried out in this case, because from history, physical examination and investigations supported a diagnosis of condylomata acuminate.

In this case, we also do PCR examination to know the type of HPV can caused condylomata acuminate in this patient. The result of PCR examination of this patient were HPV type $6,11,16$ and 18 positive. It means that, in this patient both types of HPV were found, namely low risk and high risk types, but for the high risk type of HPV, HPV type 16 and 18, this type is known could cause malignancy in the anogenital region including cervical cancer and anal cancer. ${ }^{1,2}$

Until now, around $100 \mathrm{HPV}$ genotypes have been known. But not all can cause genital condylomata acuminata, most commonly $70-100 \%$ of cases caused by types 6 and 11 found in the genital area and anus and types 16 and 18 which are potentially oncogenic and most often found in cervical cancer. ${ }^{1}$

There are several therapeutic modalities that can be used in condylomata acuminata including topical therapy, chemical surgery, electrical surgery, cryosurgery and excision surgery taking into account clinical manifestations, location, therapeutic complications, condition of the patient, therapeutic availability and doctor's skills or experience ${ }^{1}$

The Center for Disease Control and Prevention (CDC) provides a therapeutic choice guideline based on the anatomical location of the warts. In the vagina, the choice of therapy is cryosurgery with Nitrogen liquid and TCA. In the meatus urethral, the choice of treatment is cryosurgery with Nitrogen liquid and podofilin $10-25 \%$. At Anus, the choice of treatment is by cryosurgery with Nitrogen liquid, TCA $80-90 \%$ and excision surgery. ${ }^{3}$

Trichlorosetic acid (TCA) has a concentration that varies between $80-90 \%$. This material can penetrate quickly and has a caustic effect by giving rise to 
coagulation and necrosis in superficial tissues. The advantage of TCA, that it is very effective for small lesions. In addition, systemic absorption is low so it is safe to use in the vaginal, anal and cervical area and is safe for pregnant women. Trichloroacetic acid is applied carefully to lesions to form white frosting, applied every week and can be repeated up to a maximum of 6 weeks. Treatment success rates range from 70 to $80 \% .9$

In this patient, for the first treatment we did a chemosurgery with $80 \%$ TCA for the treatment of her condylomata acuminata. After two times treatment with $80 \%$ TCA, the warts in perineal has been released but in the place of the warts appear erotion and ulceration with pussy.

Beside that we treated this patient with cryosurgery, 3 days after cryosurgery appear a blister in the warts and the blister persist until 10 days after cryosurgery. In patiens control 17 day after cryosurgery, the warts in the upper side of labia mayora sinistra have mostly fallen out and one of the genital warts persists and does not release but in the lower side of labia mayora sinistra area was persist and no changes.

And then 2 week later, there were appear a new warts in pubis and both of groin. It could caused by history of frequent scratching the warts because of itching. For the warts, we treated with other modality of theraupic namely electrosurgery or electrocauter.

There are many modality treatment for condylomata acuminata, in this patient we treated the patient with three different modality, and the result of every modality are good result.

Recurrence after treatment often occurs especially in patients with immunocompromised. A study showed that recurrence of genital and anogenital warts was found to be $12.9 \%$ in people with HIV infection compared to patients without HIV infection of $9.3 \%$. In cases with type 16 and $18 \mathrm{HPV}$ that are positive, potentially oncogenic and most often can cause cervical cancer. Therefore, early detection and management is very important, especially in high risk populations such as do routine pap smears and vaccinations. ${ }^{9}$

\section{Conclusion}

We presented a Case of Genital and Perineal
Condylomata Acuminata in Adolescent Girl 19th Years Old with Type of HPV Low Risk and High Risk. Diagnosis in this case was made based on anamnesis, physical examination, laboratory examination and PCR examination. Patient is a student at the age of 19 years old, unmarried but has had free sex or sexual intercouse with her boyfriend. The younger a person when she first has sexual intercourse will increase a person's risk of contracting HPV infection The results of PCR examination of this patient were HPV types $6,11,16$ and 18 positive. It means that one of the types of HPV found are types 16 and 18 where this type is known to cause malignancy in the anogenital region including cervical cancer and anal cancer.

In cases with type 16 and 18 HPV that are positive, potentially oncogenic and most often can cause cervical cancer. Therefore, early detection and management is very important, especially in high risk populations such as do routine pap smears and vaccinations. There are many modality treatment for condylomata acuminata, in this patient we treated the patient with three different modality, and the result of every modality are good result.

\section{References}

1. Winer RL, Koutsky LA. Genital Human papillomavirus infection. Dalam: Holmes KK, Mardh PA, Spad ing PF et al. . Sexually transmitted diseases 4TH ed. New York: McGrawHill, 2008: 489-501.

2. Graziottin A1, Serafini A. HPV infection in women: psychosexual impact of genital wartss and intraepithelial lesions. J Sex Med. 2009 Mar; 6(3): 633-45.

3. Sanjosé S, Palefsky J. Cervical and anal HPV infections in HIV positive women and men. Virus Research. 2002; 89: 201-11.

4. Palefsky JM. Cervical human papillomavirus infection and cervical intraepithelial neoplasia in woman positive for human immunodeficiency virus in the era of highly active antirretroviral therapy. Curr Op Oncol. 2003; 15: 382-8.

5. Palefsky JM. HPV infection and HPV-associated 
neoplasia in immunocompromised women. Int $\mathrm{J}$ Gynaecol Obstet. 2006; 94(1): 6-64.

6. Muñoz N, Castellsagué X, González AB, Gissmann L. Chapter 1: HPV in the etiology of human cancer. Vaccine. 2006; 24(3): 1-10.

7. Low 1 AJ, Clayton T, Konate I, Nagot N, Ouedraogo A, Huet C, et al. Genital wartss and infection with human immunodeficiency virus in high-risk women in Burkina Faso: a longitudinal study.
BMC Infectious Diseases. 2011; 11: 20.

8. Ghaemmaghami F, Nazari Z, Mehrdad N. Female genital wartss. Asian Pacific J Cancer Prev, 8: 339347.

9. Centers for Disease Control and Prevention. [Sexually Transmitted Diseases Treatment Guidelines, 2010]. MMWR 2010; 59(No. RR12):70-3. 